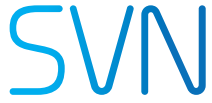

Stroke \& Vascular Neurology

\title{
Carotid artery endarterectomy in patients with symptomatic non-stenotic carotid artery disease
}

\author{
Valentina Nardi (D) ,' John C Benson, ${ }^{2}$ Anthony S Larson (D) , ${ }^{2}$ Waleed Brinjikji, ${ }^{2}$ \\ Luca Saba, ${ }^{3}$ Fredric B Meyer, ${ }^{4}$ Giuseppe Lanzino, ${ }^{4}$ Amir Lerman (D) , \\ Luis E Savastano ${ }^{4}$
}

To cite: Nardi V, Benson JC, Larson AS, et al. Carotid artery endarterectomy in patients with symptomatic non-stenotic carotid artery disease. Stroke \& Vascular Neurology 2022;0. doi:10.1136/svn-2021-000939

- Additional supplemental material is published online only. To view, please visit the journal online (http://dx.doi.org/10. 1136/svn-2021-000939).

Received 13 February 2021 Accepted 8 April 2021

\section{Check for updates}

(c) Author(s) (or their employer(s)) 2022. Re-use permitted under CC BY-NC. No commercial re-use. See rights and permissions. Published by BMJ.

${ }^{1}$ Department of Cardiovascular Diseases, Mayo Clinic, Rochester, Minnesota, USA ${ }^{2}$ Radiology, Mayo Clinic, Rochester, Minnesota, USA ${ }^{3}$ Radiology, Azienda Ospedaliero Universitaria, Cagliari, Italy ${ }^{4}$ Neurosurgery, Mayo Clinic, Rochester, Minnesota, USA

Correspondence to Dr Luis E Savastano; Savastano.luis@mayo.edu

\section{ABSTRACT}

Object We sought to determine the safety and efficacy in secondary stroke prevention of carotid endarterectomy (CEA) in patients with symptomatic non-stenotic carotid artery disease (SyNC).

Methods This was a single-centre retrospective case series. All patients who underwent CEA for unilateral anterior circulation cerebrovascular events with ipsilateral $<50 \%$ carotid stenosis from 2002 to 2020 were included. Imaging hallmarks including the degree of luminal stenosis and the presence of various vulnerable plaque characteristics (eg, intraplaque haemorrhage (IPH) on MR angiography, ulceration or low-density plaque on CT angiography) were assessed. The presence of vulnerable plaque characteristics was compared between arteries ipsilateral to the ischaemic event and contralateral arteries. The prevalence of perioperative/intraoperative complications, as well as recurrent ischaemic events at follow-up was determined.

Results Thirty-two patients were included in the analysis, of which $25.0 \%$ were female. Carotid arteries ipsilateral to an ischaemic event had a significantly higher prevalence of IPH when compared with contralateral arteries $(80.0 \%$ vs $0.0 \% ; p<0.001)$. There were no intraoperative complications. One patient (3.1\%) developed symptoms of transient ipsilateral ischaemia 1 day following CEA which resolved without treatment. In a median follow-up of 18.0 months (IQR 5.0-36.0), only one patient (3.1\%) experienced a transient neurologic deficit with complete resolution (annualised rate of recurrent stroke after CEA of $1.5 \%$ for a total follow-up of 788 patient-months following CEA). All other patients $(31 / 32,96.9 \%)$ were free of recurrent ischaemic events.

Conclusion CEA appears to be safe and well-tolerated in patients with SyNC. Additional studies with larger cohorts and longer follow-up intervals are needed in order to determine the role of CEA in this patient population.

\section{INTRODUCTION}

The management of patients with carotid artery disease is well defined in evidencebased guidelines which consider the degree of luminal stenosis as the main criteria as an indication for intervention. ${ }^{1}$ The two main trials which provided evidence were the North American Symptomatic Carotid Endarterectomy Trial (NASCET), ${ }^{2}$ and the
European Carotid Surgery Trial. ${ }^{3}$ These large, randomised clinical trials, conducted more than three decades ago, risk stratified patients by the degree of lumen narrowing on catheter angiography. Based on these landmark studies, the management of carotid artery disease was influenced in two ways. On the one hand, these trials showed that carotid endarterectomy (CEA) significantly reduces the risk of recurrent stroke compared with medical therapy alone in symptomatic patients with moderate $(50 \%-69 \%)$ and severe $(>70 \%)$ stenosis. ${ }^{23}$ On the other hand, the degree of luminal stenosis became the primary surrogate for carotid-related stroke risk and indication for revascularisation. Consequently, patients with documented carotid atherosclerotic disease ipsilateral to an ischaemic stroke who did not reach the $50 \%$ stenosis limit were managed medically without revascularisation.

Recent biological and clinical data have demonstrated that pathological changes within the vessel wall determine the fate of a plaque regardless of the severity of stenosis. ${ }^{4}$ By extension, structural and biological factors that define plaque stability and vulnerability, especially at the lumen/wall interface, might be more significant than the degree of narrowing in determining the risk profile and root cause of a cerebrovascular event, even in patients with mild $(<50 \%)$ carotid stenosis. ${ }^{4-7}$ However, current guidelines suggest that carotid revascularisation should not be pursued in this patient population.

Over the years at our institution, we have performed CEA in a series of highly selected patients with mild stenosis and cerebrovascular ischaemic events which are often recurrent despite intensive medical management. These patients typically possess imaging features of plaque vulnerability and mild $(<50 \%)$ luminal stenosis within the ipsilateral carotid artery without any other identifiable embolic source. Such patients may 
be said to have symptomatic non-stenotic carotid artery disease (SyNC), or SyNC as defined by Goyal et al. ${ }^{8}$ If this management paradigm is safe and efficacious, it may call into question the emphasis that current guidelines place exclusively on the degree of luminal stenosis without consideration of plaque morphological characteristics as seen on imaging. The objective of the current study was to therefore describe our institutional cohort of patients with ischaemic cerebral events who underwent CEA for SyNC.

\section{METHODS}

\section{Patient selection}

We performed a retrospective analysis of a prospectively maintained clinical database of consecutive patients who underwent CEA at the Department of Neurosurgery at a single institution between November 2002 and March 2020. We included patients that met the following criteria: (1) history of at least one cerebrovascular event involving the anterior circulation (ischaemic stroke, transient ischaemic attack (TIA) or amaurosis fugax); (2) standard diagnostic stroke workup including transthoracic echocardiography and continuous ECG monitoring for at least 24 hours which was negative for any cause of the patient's ischaemic event; (3) brain and carotid vascular imaging with CT angiography (CTA) and/or MR angiography (MRA) with or without vessel wall imaging (VWI) showing ipsilateral carotid luminal stenosis of $<50 \%$ as defined by NASCET criteria. Patients with 50\% stenosis or higher were excluded. SyNC was considered a non-lacunar stroke involving only one anterior circulation territory, negative trans-thoracic cardiac echocardiogram and at least 24 hours of cardiac ECG monitoring, $<50 \%$ stenosis in the ipsilateral carotid by NASCET criteria along with high-risk imaging features and no other specific cause of stroke identified.

Next, the following data were extracted from the medical records of each included patient: demographic characteristics, cardiovascular risk factors, medical treatment, type and number of cerebrovascular and cardiovascular events, radiologic findings, surgical features and follow-up data.

\section{Radiological evaluation}

All patients underwent brain and neck CTA and/or MRA and/or MR VWI during presurgical evaluation. CTA was performed on a Siemens single or dual source CT scanner. MRA images were recorded on a 3T MRI scanner (GE 750, GE Healthcare, Milwaukee, Wisconsin, USA) with a 16-channel head/neck/spine coil covering the head and the neck. CTA and MRA were both used to measure the severity of stenosis of the carotid arteries on axial imaging as a percentage of lumen narrowing as defined by NASCET criteria. A non-stenotic artery (or mild stenosis) was defined as less than $50 \%$ stenosis. CTA was used to evaluate for the presence of plaque features such as low-density plaque (LDP) components (defined as hypodensity within the plaque $<50$ Hounsfield units), calcification (hyperdensity within the plaque $>120$ Hounsfield units) and ulceration (defined as a luminal defect or indentation of the plaque surface $>1000 \mu \mathrm{m}$ ). Intraplaque haemorrhage (IPH) was assessed on MR using T1-weighted magnetisation-prepared rapid acquisition with gradient echo (MPRAGE) sequences in the coronal plane when available. All markers of vulnerability were defined according to previously described criteria. ${ }^{479}$ The analysis of preoperative imaging including quantification of the degree of stenosis and biomarkers of vulnerability was performed by a board-certified neuroradiologist (JB) blinded to patient information. The degree of stenosis and presence of vulnerable plaque features were compared between symptomatic carotid arteries and the contralateral carotid in each patient.

\section{Surgical treatment}

Carotid revascularisation in the setting of SyNC was performed after informed consent, usually in the case of recurrent ischaemic cerebrovascular events despite maximal medical management (which included dual antiplatelet therapy, statins, etc) and/or findings of plaque vulnerability (such as IPH, LDP, ulceration) on imaging. Two senior neurosurgeons (GL and FM) performed all the surgical procedures under general anaesthesia. Routine intraoperative electroencephalography monitoring for selective intraluminal shunting was used. ${ }^{10}$ Follow-up information following CEA was collected from a review of medical records or by telephone survey.

\section{Statistical Analysis}

Statistical analyses were performed using JMP Pro software (SAS Institute). Baseline characteristics of the study population were presented as mean $\pm \mathrm{SD}$ for normally distributed continuous variables and median and IQR when not normally distributed. Categorical variables were expressed as frequency (percentage). Comparison of ipsilateral to contralateral degree of stenosis was made with a paired Student's t-test. Comparison of prevalence of plaque features ipsilateral and contralateral to stroke was made with McNemar test. P values of less than 0.05 were accepted as significant.

\section{RESULTS}

\section{Demographic and clinical data}

Thirty-two patients were included in the final study cohort. The median age was 72.0 years (IQR 65.5-76.0) and $25.0 \%$ were female. The most common clinical presentations were ischaemic stroke (18/32 patients, 56.2\%) and TIA $(9 / 32,28.1 \%)$. Amaurosis fugax was present in a smaller proportion of patients $(5 / 32,15.6 \%)$. Twentythree patients $(71.8 \%)$ had recurrent ipsilateral cerebrovascular events with a median number of four episodes per patient (IQR 3-5) prior to CEA despite medical management that included at least antiplatelet therapy. Of note, a few patients $(7,21.9 \%)$ were not on statin therapy at the time of CEA: 5 were statin intolerant due to side effects, 
Table 1 Presurgical neuroimaging findings

\begin{tabular}{llll} 
& Ipsilateral carotid artery & Contralateral carotid artery & P value \\
\hline No of carotids & 32 & 32 & \\
Stenosis \% & $30.0(20.0-40.0)$ & $22.5(10.0-50.0)$ & \\
Range \% of stenosis & $0.0-45.0$ & $0.0-99.0$ & $<0.001$ \\
Ipsilateral IPH & $12(80.0)$ & $0(0.0)$ & \\
Plaque vulnerability features on CTA-no (\%) & & & 0.157 \\
$\quad$ LDP & $13(61.9)$ & $9(42.8)$ & 0.414 \\
$\quad$ Ulceration & $4(19.0)$ & $2(9.5)$ & 1.000 \\
$\quad$ Calcification & $20(95.2)$ & $20(95.2)$ &
\end{tabular}

Values are presented as means (SD) or median (IQR) for continuous variables and percentages for dichotomous or categorical variables. CTA, CT angiography; IPH, intraplaque haemorrhage; LDP, low-density plaque components.

and two had lipid profiles levels already at goal. Details of baseline characteristics, medical history and therapy at the presurgical clinical evaluation are summarised in online supplemental table 1.

\section{Relationship between plaque features and degree of stenosis}

Preoperative imaging findings in the carotid arteries ipsilateral and contralateral to the ischaemic events are outlined in table 1 . Vulnerability plaque features on CTA and MRA imaging are demonstrated in figure 1. CTA was performed in 21/32 patients $(65.6 \%)$, MRA and/or MR VWI in 30/32 patients (93.7\%) and MR with MPRAGE in $15 / 32$ patients $(46.8 \%)$. Symptomatic plaques were on the left side in 23/32 patients (71.8\%). The median degree of arterial stenosis ipsilateral to the ischaemic event was 30.0 (IQR 20.0-40.0) compared with 22.5 (IQR 10.0-50.0) on the contralateral side. There was no correlation between the presence of IPH and the degree of stenosis ipsilateral to the ischaemic event $(p=0.601)$. Seven patients $(21.9 \%)$ showed LDP in bilateral carotids. There was no correlation between the presence of LDP and the degree of stenosis ipsilateral

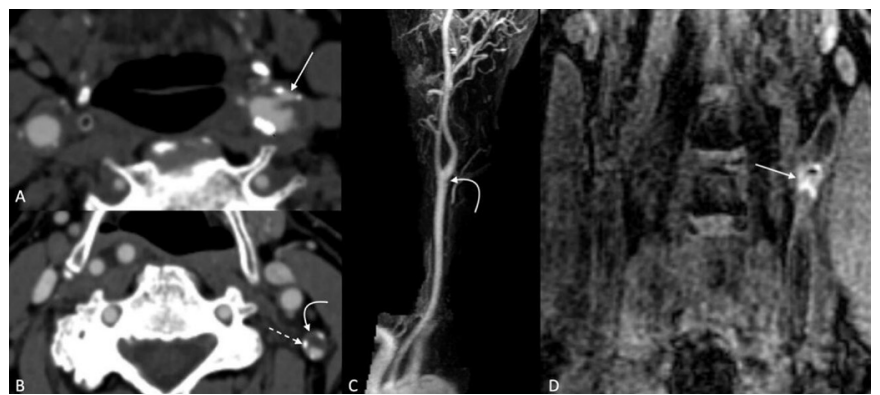

Figure 1 Examples of vulnerability plaque features on CTA and MRA imaging. Ulcerations (A) appear as focal defects in the plaque that may be regular or irregular (arrow). Low-density plaque components (curved arrow), (B) are notably hypodense, and are often surrounded by peripheral calcifications (dashed arrow). Even plaques that cause minimal luminal narrowing (arrow), (C) may demonstrate large intraplaque haemorrhage on coronal MPRAGE images (D). CTA, CT angiography; MPRAGE, magnetisation-prepared rapid acquisition with gradient echo; MRA, MR angiography. to the ischaemic event $(\mathrm{p}=0.107)$. There was no correlation between the presence of ulceration and the degree of stenosis ipsilateral to the ischaemic event $(\mathrm{p}=0.447)$. Twenty patients $(95.2 \%)$ had bilateral carotid calcification $(p=1.000)$, with no significant correlation with the lumen narrowing percentage $(\mathrm{p}=0.08)$.

\section{Relationship between plaque features and ipsilateral ischaemic events}

Twelve patients $(80.0 \%)$ out of the 15 patients who underwent MR with MPRAGE were found to have an IPH in the ipsilateral carotid artery compared with none in the contralateral artery $(\mathrm{p}<0.001)$. LDP on CTA was found in 13/21 of the ipsilateral carotids and 9/21 of the contralateral carotids ( $61.9 \%$ vs $42.8 \%$; $\mathrm{p}=0.157)$. Ulceration was identified in $19.0 \%$ of the ipsilateral carotids vs $9.5 \%$ of the contralateral carotids $(\mathrm{p}=0.414)$.

\section{Intraoperative and postoperative course}

All 32 patients with SyNC underwent CEA without any intraoperative complications. Postoperatively (within 30 days), one patient on a dual antiplatelet regimen developed a haematoma in the operative bed requiring washout. One patient $(3.1 \%)$ had a transient neurologic deficit consisting of expressive aphasia (which was the initial presenting symptom) a few hours after CEA which was likely triggered by orthostatic hypotension and resolved after $10 \mathrm{~min}$ of recumbency and fluids. None experienced any ischaemic or haemorrhagic stroke, infection, wound issues (other than the haematoma described above), hyperperfusion syndrome, dysphagia lasting $>1$ min or death within 30 days following CEA (table 2). A patient case example is demonstrated in figure 2.

\section{Follow-up and long-term outcomes}

The median follow-up after surgery was 18.0 months (IQR 5.0-36.0) with a total follow-up of 788 patientmonths. One patient $(3.1 \%)$ presented with a possible TIA 7.8 years after CEA, although subsequent work up revealed no evidence of atheroma, thrombus or stenosis at the post-surgical carotid bifurcation. The remainder of patients $(31 / 32,96.9 \%)$ did not have any recurrent 
Table 2 Complications and cardiovascular events in the follow-up to CEA

\begin{tabular}{ll}
\hline & $\begin{array}{l}\text { Carotid mild (<50\%) } \\
\text { stenosis (N=32) }\end{array}$ \\
\hline Perisurgical complications -no (\%) & $0(0.0)$ \\
\hline Postsurgical complications-no (\%) & $2(6.2)$ \\
\hline Haematoma & $1(3.1)$ \\
\hline Transitory neurological deficit & $1(3.1)$ \\
\hline Hyper-perfusion syndrome & $0(0.0)$ \\
\hline Median follow-up-months, (IQR) & $18.0(5.0-36.0)$ \\
\hline $\begin{array}{l}\text { Follow-up >1 year-no (\%) } \\
\text { Cardiovascular events in follow-up }\end{array}$ & $20(62.5)$ \\
\hline $\begin{array}{l}\text { Ischaemic stroke-no (\%) } \\
\text { TIA-no (\%) }\end{array}$ & $0.0(0.0)$ \\
\hline $\begin{array}{l}\text { Amaurosis fugax-no (\%) } \\
\text { Recurrent ischaemic cerebrovascular } \\
\text { events related to ipsilateral carotid } \\
\text { disease-no (\%) }\end{array}$ & $0(0.0)$ \\
\hline Deaths-no (\%) & $3(9.3)$ \\
\hline
\end{tabular}

Values are presented as means (SD) or median (IQR) for continuous variables and percentages for dichotomous or categorical variables.

CEA, carotid endarterectomy; TIA, transient ischaemic attack.

ipsilateral ischaemic events at follow-up. There were three late deaths that occurred 7 years or later after CEA. The causes of death in these patients were cancer and complications from peripheral vascular disease. Operative and follow-up data are summarised in table 2.

\section{DISCUSSION}

To our knowledge, the current study presents the largest case series of CEA performed for SyNC. In this singlecentre cohort, carotid revascularisation was performed in a highly selected group of patient with a negative cardioembolic workup and contemporary high-risk vulnerability features on plaque imaging. Most of the patients underwent surgery after multiple recurrent ipsilateral ischaemic cerebrovascular events despite intensive medical management. Carotid revascularisation was conducted without significant complications and resulted in excellent secondary stroke prevention with virtually no carotid-related recurrent ischaemic events at follow-up. These data strengthen an etiological role of SyNC in thromboembolic strokes. Furthermore, our findings may demonstrate the need to revise the role of carotid revascularisation in patients with mild ipsilateral stenosis and imaging features of plaque vulnerability by prospective trials.

Arterial stenosis leads to increasing haemodynamic stress and is an established factor that statistically correlates with higher likelihood of having future ischaemic events. ${ }^{11}$ However, the classical paradigm focusing on stenosis has changed and it is now broadly accepted that the vast majority of cerebrovascular events are due to acute thromboembolic events driven by plaque rupture. ${ }^{4}$ By consequence, a symptomatic plaque need not be large enough to cause a certain degree of stenosis, but 'vulnerable' which may result in rupture, thrombosis and distal embolisation causing stroke. ${ }^{12}$ Despite the major advances in our understanding of carotid atherosclerosis physiopathology, the clinical management has lagged behind as the selection of patients with carotid artery disease for carotid revascularisation continues to rely on the degree of stenosis alone. Based on multiple randomised controlled trials conducted in the $80 \mathrm{~s}$ and 90 s and using catheter angiography as the gold standard imaging tool for evaluation of carotid artery disease, ${ }^{2} 313-15$ CEA is supported for secondary stroke prevention in moderate $(50 \%-69 \%)$ and severe $(\geq 70 \%)$. In cases of mild stenosis $(<50 \%)$ CEA is currently not recommended by published guidelines given lack of additional benefit from CEA relative to conservative management alone. However, subjects in these studies who underwent CEA were heterogeneous and only a small percentage may have truly had SyNC. This limitation may have led to a dilution of any potential benefits of the intervention. Despite current recommendations, clinical experience has repeatedly shown the inadequacy of limiting the assessment of atherosclerosis disease to the degree of stenosis alone, and therefore, the need to better understand biomarkers of plaque vulnerability based on imaging has gained attention. It is now accepted that factors such as ulceration, hypodense plaque on CT and plaque haemorrhage on MR, ${ }^{4916-18}$ which may all indicate structural instability at the lumen/wall interface, are associated with a higher

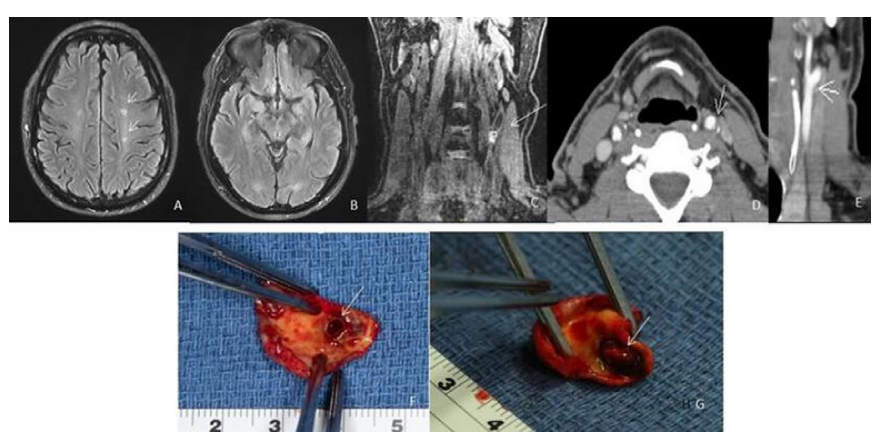

Figure 2 Case example of a patient in our cohort with recurrent strokes and $<50 \%$ stenosis of carotid artery. (A, B) Evidence of ischaemic areas on preoperative MRA (arrows); (C) intraplaque haemorrhage on MRA, MPRAGE sequence (arrow); (D, E) stenosis $<50 \%$ and ulceration of the fibrous cap on CTA (arrows); intraoperative observation of the carotid plaque removed en block during carotid endarterectomy before squeezing $(F)$ and after squeezing $(\mathrm{G})$. The specimen presents a large ulceration (arrows) on the surface that enables the egress of necrotic material and blood to the luminal surface with squeezing. CTA, CT angiography; MPRAGE, magnetiation-prepared rapid acquisition with gradient echo; sMRA, MR angiography. 
risk of embolic events, even if the lumen has a low-grade narrowing.

In our cohort, among the 15 patients that had preoperative MR with MPRAGE, ${ }^{17} 12(80 \%)$ had IPH in the carotid artery ipsilateral to the ischaemic event, while none showed IPH in the contralateral carotid artery. These data support the hypothesis of a mechanistic role of IPH in the pathogenesis of stroke and/or IPH is a byproduct of plaque disruption. As reported by previous authors, IPH within carotid plaque has been associated with ipsilateral ischaemic events. ${ }^{19-21}$ Thus, it may be considered a marker of plaque vulnerability. Moreover, according to our results based on CTA imaging, carotid plaques ipsilateral to symptoms were more likely to have LDP components and ulceration compared with the contralateral side, although the difference was not significant. Both of these plaque findings are known markers of plaque vulnerability. ${ }^{49}$ Interestingly, calcification was the most common finding on CTA and equally represented in both sides. The role of calcium in the vessel wall of atherosclerotic carotid artery is controversial and has not been fully elucidated as of yet. In some studies carotid plaque calcification is considered as a protective plaque feature associated with plaque stability, ${ }^{22}{ }^{23}$ while other authors have recently opined that calcium within the carotid plaque is a marker of instability. ${ }^{24}$

Moreover, in our cohort, there were no significant operative complications and no recurrent strokes attributed to the revascularised carotid with a total of 788 patient-months of follow-up. This strongly supports an etiological role of the ipsilateral non-stenotic plaque as a root cause of stroke and suggests that carotid revascularisation could be a valuable option in this patient population. This study supports the hypothesis that the selection of patients based on the mere degree of stenosis may not be optimal, and surgery should not be precluded merely if an arbitrary threshold is not reached in symptomatic patients with imaging features of plaque vulnerability. Recent publications have shown an increased risk of ipsilateral recurrent strokes refractory to medical treatment in a subset of patients with mild carotid artery stenosis and imaging features of plaque vulnerability. ${ }^{25-28}$

Our group has recently published a case series of patients (from the same institution as the cohort included in this study) with symptomatic non-stenotic haemorrhagic plaques that were medically managed and found a $9.5 \%$ annual rate of recurrent stroke (42 patient-years of follow-up). ${ }^{29}$ These patients had an annualised rate of recurrent stroke after CEA of $1.5 \%$ (66 patient-years of follow-up). Additional, recent studies with robust sample sizes have demonstrated an association between ischaemic cerebral events and ipsilateral SyNC, particularly in plaques with vulnerable features. ${ }^{3031}$ Taken together, these data infer that guidelines should not be considered as strict protocols and interventional decisions should not be based solely on the degree of luminal stenosis. Individual characteristics of a patient and imaging features of plaque vulnerability should be taken into account: a stenosis of less than $50 \%$ should not disqualify a patient from consideration for CEA. Future research focused on defining robust biomarkers of plaque vulnerability and their use in risk stratification in prospective randomised clinical trials is largely needed.

Intriguingly, Goyal et al have recently suggested working definitions of SyNC, given that a diagnosis of cryptogenic stroke likely precludes patients from undergoing targeted stroke preventative therapy. In other words, if patients were to meet certain criteria for subtypes of SyNC (definite, likely or probable SyNC based on the presence of various factors), then a targeted approach may be pursued for secondary prevention. Although SyNC represents a plausible aetiology of ipsilateral stroke, confirmatory studies are needed to confirm SyNC as a definite stroke aetiology before any targeted therapy can be evaluated. Whether or not CEA would be a efficacious modality for secondary prevention in such cases requires further evaluation.

Our work indicates that CEA is a safe and well-tolerated intervention in patients with SyNC in the absence of another plausible stroke source. Although it may be that CEA prevents future ischaemic events in such patients, prospective clinical trials are largely needed. This work also suggests that a subpopulation of patients having embolic strokes of unknown aetiology with ipsilateral substenotic carotid disease could harbour thrombogenic lesions in non-stenosing complicated plaques. Therefore, dedicated carotid wall imaging capable to identify plaque disruption at the lumen/wall interface and not just luminal narrowing measurement may be a beneficial component in the workup of patients with embolic strokes of an unknown aetiology. ${ }^{32} 33$

In addition to the relevance in stroke aetiology, growing clinical evidence has shown an increased risk of ipsilateral recurrent strokes refractory to medical treatment in a subset of patients with SyNC and vulnerable plaque features, in particular IPH. ${ }^{25} 2734$ This pathological hallmark of plaque instability is important as current intensive medical management, especially in patients with recurrent strokes, includes dual antiplatelet therapy and in cases of embolic strokes of unknown source, which may paradoxically worsen plaque haemorrhage. ${ }^{35}$ In our cohort, $93.7 \%$ of patients were on aspirin and half of the patients were also on clopidogrel prior to the intervention, with $71.8 \%$ of patients having recurrent ipsilateral cerebrovascular events. Clinically, the management of symptomatic patients with mild carotid stenosis remains challenging. However, CEA may be a valid and an effective therapeutic option as supported by other single centre surgical series. ${ }^{528}$ 36-38

As mentioned, current guidelines do not recommend the use of CEA in cases of $>50 \%$ carotid stenosis. This recommendation, however, is based on the degree of luminal stenosis and does not take into account whether or not radiographic evidence of vulnerable plaque is 
present. Indeed, a growing body of data suggests that SyNC is likely associated with ipsilateral stroke, although more definitive studies are necessary. ${ }^{81} 3031$ In patients with SyNC and no other obvious source of stroke, CEA may represent a targeted option for secondary prevention, though this has yet to be confirmed.

Our study has limitations. First, this is a relatively small observational case series of 32 highly selected patients analysed by retrospective analysis of data from a single institution. The selection bias is further amplified by the lack of control group and patient randomisation, and therefore, we are unable to draw any robust conclusions regarding the efficacy of CEA in context of SyNC. The median follow-up period of 18 months is a relatively short interval, and longer follow-up times are needed in order to evaluate for recurrent ipsilateral ischaemic events. However, most recurrent cerebrovascular events are known to recur within few months (even a few weeks) from the qualifying events, and therefore we think that our follow-up is representative. Moreover, taking into consideration that our cohort was formed by $>70 \%$ of patients with recurrent strokes despite intensive medical management, an annualised rate of recurrent stroke after CEA of $1.5 \%$ for a total follow-up of 788 patient-months is likely clinically significant. Despite the limitations, to our knowledge, this is the largest surgical series of patients with SyNC employing contemporary imaging features of vulnerability reported to date. Thus, this study may serve as preliminary data for further clinical trials using contemporary biomarkers of plaque vulnerability to risk stratify patients.

\section{CONCLUSIONS}

CEA appears to be safe and well tolerated in patients with symptomatic non-stenotic carotid stenosis and imaging markers of plaque vulnerability. Prospective randomised trials employing imaging biomarkers of plaque instability are needed to define the role of carotid revascularisation in this patient population.

Contributors VN: data gathering, data analysis, draft writing, draft editing. JB: data gathering, data analysis, draft editing. AL: data gathering, data analysis, draft editing, submission. WB: concept design, data analysis, draft editing. LS: data analysis, draft editing. FM: data gathering, data analysis, draft editing. GL: concept design, data gathering, data analysis, draft editing. AL: concept design, data gathering, data analysis, draft editing. LS: concept design, data gathering, data analysis, draft editing, guarantor.

Funding The authors have not declared a specific grant for this research from any funding agency in the public, commercial or not-for-profit sectors.

Competing interests None declared.

Patient consent for publication Not applicable.

Ethics approval All patients included in this study provided written informed consent for participation in research activities at our institution. This study was approved by Mayo Clinic Institutional Review Board.

Provenance and peer review Not commissioned; externally peer reviewed.

Data availability statement Data are available on reasonable request. All data are available on reasonable request. Please contact VN, nardi.valentina@mayo.edu.
Open access This is an open access article distributed in accordance with the Creative Commons Attribution Non Commercial (CC BY-NC 4.0) license, which permits others to distribute, remix, adapt, build upon this work non-commercially, and license their derivative works on different terms, provided the original work is properly cited, appropriate credit is given, any changes made indicated, and the use is non-commercial. See: http://creativecommons.org/licenses/by-nc/4.0/.

\section{ORCID iDs}

Valentina Nardi http://orcid.org/0000-0003-4601-0434

Anthony S Larson http://orcid.org/0000-0001-6021-3452

Amir Lerman http://orcid.org/0000-0002-9446-5313

\section{REFERENCES}

1 Savastano LE, Seibel EJ. Scanning fiber angioscopy: a multimodal intravascular imaging platform for carotid atherosclerosis. Neurosurgery 2017;64:188-98.

2 North American Symptomatic Carotid Endarterectomy Trial Collaborators, Barnett HJM, Taylor DW, et al. Beneficial effect of carotid endarterectomy in symptomatic patients with high-grade carotid stenosis. N Engl J Med 1991;325:445-53.

3 Randomised trial of endarterectomy for recently symptomatic carotid stenosis: final results of the MRC European carotid surgery trial (ECST). The Lancet 1998;351:1379-87.

4 Brinjikji W, Huston J, Rabinstein AA, et al. Contemporary carotid imaging: from degree of stenosis to plaque vulnerability. J Neurosurg 2016;124:27-42.

5 Kashiwazaki D, Shiraishi K, Yamamoto S, et al. Efficacy of Carotid Endarterectomy for Mild (<50\%) Symptomatic Carotid Stenosis with Unstable Plaque. World Neurosurg 2019;121:e60-9.

6 Gates MJ, Brinjikji W, Williams L, et al. Mild carotid stenosis with recurrent symptoms triggered by eating. World Neurosurg 2017;97:750. e11-50. e13.

7 Saba L, Yuan C, Hatsukami TS, et al. Carotid artery wall imaging: perspective and guidelines from the ASNR vessel wall imaging study Group and expert consensus recommendations of the American Society of Neuroradiology. AJNR Am J Neuroradiol 2018;39:E9-31.

8 Goyal M, Singh N, Marko M, et al. Embolic stroke of undetermined source and symptomatic Nonstenotic carotid disease. Stroke 2020;51:1321-5

9 Saba L, Saam T, Jäger HR, et al. Imaging biomarkers of vulnerable carotid plaques for stroke risk prediction and their potential clinical implications. Lancet Neurol 2019;18:559-72.

10 Perkins WJ, Lanzino G, Brott TG. Carotid stenting vs endarterectomy: new results in perspective. Mayo Clin Proc 2010;85:1101-8.

11 Slager CJ, Wentzel JJ, Gijsen FJH, et al. The role of shear stress in the destabilization of vulnerable plaques and related therapeutic implications. Nat Clin Pract Cardiovasc Med 2005;2:456-64.

12 Howard DP, van Lammeren GW, Rothwell PM, et al. Symptomatic carotid atherosclerotic disease: correlations between plaque composition and ipsilateral stroke risk. Stroke 2015;46:182-9.

13 Hobson R, Brott T, Ferguson R. CREST: carotid revascularization endarterectomy versus stent trial. London, England: SAGE Publications Sage UK, 1997.

14 Walker MD, Marler JR, Goldstein M. Endarterectomy for asymptomatic carotid artery stenosis. JAMA 1995;273:1421-8.

15 Halliday A, Mansfield A, Marro J, et al. Prevention of disabling and fatal strokes by successful carotid endarterectomy in patients without recent neurological symptoms: randomised controlled trial. Lancet 2004;363:1491-502.

16 Seyedsaadat SM, Rizvi A, Alzuabi M, et al. Correlation of MRIdetected vulnerable carotid plaques with clinical presentation: a systematic review and meta-analysis. J Neurosurg Sci 2020;64:263-71.

17 Brinjikji W, DeMarco JK, Shih R, et al. Diagnostic accuracy of a clinical carotid plaque MR protocol using a neurovascular coil compared to a surface coil protocol. J Magn Reson Imaging 2018:48:1264-72.

18 Saba L, Moody AR, Saam T, et al. Vessel Wall-Imaging Biomarkers of Carotid Plaque Vulnerability in Stroke Prevention Trials: A viewpoint from The Carotid Imaging Consensus Group. JACC Cardiovasc Imaging 2020;13:2445-56.

19 Hosseini AA, Kandiyil N, Macsweeney STS, et al. Carotid plaque hemorrhage on magnetic resonance imaging strongly predicts recurrent ischemia and stroke. Ann Neurol 2013;73:774-84.

20 Yamada K, Yoshimura S, Shirakawa M, et al. Asymptomatic moderate carotid artery stenosis with intraplaque hemorrhage: 
Progression of degree of stenosis and new ischemic stroke. $J$ Clin Neurosci 2019;63:95-9.

21 Schindler A, Schinner R, Altaf N, et al. Prediction of stroke risk by detection of hemorrhage in carotid plaques: meta-analysis of individual patient data. JACC Cardiovasc Imaging 2020;13:395-406.

22 Baradaran H, Al-Dasuqi K, Knight-Greenfield A, et al. Association between carotid plaque features on cta and cerebrovascular ischemia: a systematic review and meta-analysis. AJNR Am J Neuroradiol 2017;38:2321-6.

23 Nandalur KR, Hardie AD, Raghavan P, et al. Composition of the stable carotid plaque: insights from a multidetector computed tomography study of plaque volume. Stroke 2007;38:935-40.

24 Yoon WJ, Crisostomo P, Halandras P, et al. The use of the Agatston calcium score in predicting carotid plaque vulnerability. Ann Vasc Surg 2019;54:22-6.

25 Altaf N, Daniels L, Morgan PS, et al. Detection of intraplaque hemorrhage by magnetic resonance imaging in symptomatic patients with mild to moderate carotid stenosis predicts recurrent neurological events. J Vasc Surg 2008;47:337-42.

26 Yoshida K, Fukumitsu R, Kurosaki Y, et al. The association between expansive arterial remodeling detected by high-resolution MRI in carotid artery stenosis and clinical presentation. $J$ Neurosurg 2015;123:434-40.

27 Karlsson L, Kangefjärd E, Hermansson S, et al. Risk of Recurrent Stroke in Patients with Symptomatic Mild (20-49\% NASCET) Carotid Artery Stenosis. Eur J Vasc Endovasc Surg 2016;52:287-94.

28 Ballotta E, Angelini A, Mazzalai F, et al. Carotid endarterectomy for symptomatic low-grade carotid stenosis. J Vasc Surg 2014;59:25-31.

29 Larson AS, Nasr DM, Rizvi A, et al. Embolic stroke of undetermined source: the association with carotid intraplaque hemorrhage. JACC Cardiovasc Imaging 2021:14:506-8.
30 Singh N, Marko M, Ospel JM, et al. The risk of stroke and TIA in Nonstenotic carotid plaques: a systematic review and meta-analysis. AJNR Am J Neuroradiol 2020;41:1453-9.

31 Ospel JM, Singh N, Marko M, et al. Prevalence of ipsilateral Nonstenotic carotid plaques on computed tomography angiography in embolic stroke of undetermined source. Stroke 2020;51:1743-9.

32 Savastano LE, Zhou Q, Smith A, et al. Multimodal laser-based angioscopy for structural, chemical and biological imaging of atherosclerosis. Nat Biomed Eng 2017;1. doi:10.1038/s41551-0160023. [Epub ahead of print: 1002 2017].

33 Savastano LE, Seibel E, Wang T, et al. Abstract 107: laser angioscopy reveals concealed ulcers in Substenotic carotid plaques in cryptogenic stroke patients: a preclinical study. Stroke2017;48:A10 7-A07.

34 Yoshida K, Sadamasa N, Narumi O, et al. Symptomatic low-grade carotid stenosis with intraplaque hemorrhage and expansive arterial remodeling is associated with a high relapse rate refractory to medical treatment. Neurosurgery 2012;70:1143-51.

35 Mujaj B, Bos D, Muka T, et al. Antithrombotic treatment is associated with intraplaque haemorrhage in the atherosclerotic carotid artery: a cross-sectional analysis of the Rotterdam study. Eur Heart $J$ 2018;39:3369-76.

36 Yoshida K, Fukumitsu R, Kurosaki Y, et al. Carotid endarterectomy for medical therapy-resistant symptomatic low-grade stenosis. World Neurosurg 2019;123:e543-8.

37 Ahmed RM, Harris JP, Anderson CS, et al. Carotid endarterectomy for symptomatic, but "haemodynamically insignificant" carotid stenosis. Eur J Vasc Endovasc Surg 2010;40:475-82.

38 Takai H, Uemura J, Yagita Y, et al. Plaque characteristics of patients with symptomatic mild carotid artery stenosis. J Stroke Cerebrovasc Dis 2018;27:1930-6. 